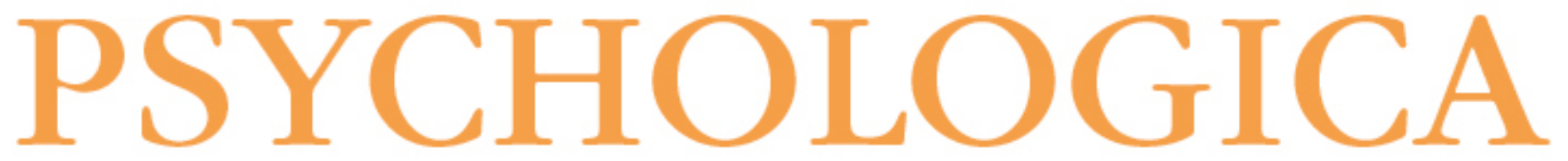

\title{
Medindo as percepções de auto-eficácia no âmbito da aprendizagem e da carreira: introdução da Secção Temática
}
Autor(es):
Silva, José M. Tomás da
Publicado por: Imprensa da Universidade de Coimbra
URL
persistente:
URI:http://hdl.handle.net/10316.2/5463
DOI:
DOI:http://dx.doi.org/10.14195/1647-8606_51_1
Accessed : $\quad$ 26-Apr-2023 11:49:05

A navegação consulta e descarregamento dos títulos inseridos nas Bibliotecas Digitais UC Digitalis, UC Pombalina e UC Impactum, pressupõem a aceitação plena e sem reservas dos Termos e Condições de Uso destas Bibliotecas Digitais, disponíveis em https://digitalis.uc.pt/pt-pt/termos.

Conforme exposto nos referidos Termos e Condições de Uso, o descarregamento de títulos de acesso restrito requer uma licença válida de autorização devendo o utilizador aceder ao(s) documento(s) a partir de um endereço de IP da instituição detentora da supramencionada licença.

Ao utilizador é apenas permitido o descarregamento para uso pessoal, pelo que o emprego do(s) título(s) descarregado(s) para outro fim, designadamente comercial, carece de autorização do respetivo autor ou editor da obra.

Na medida em que todas as obras da UC Digitalis se encontram protegidas pelo Código do Direito de Autor e Direitos Conexos e demais legislação aplicável, toda a cópia, parcial ou total, deste documento, nos casos em que é legalmente admitida, deverá conter ou fazer-se acompanhar por este aviso. 
NÚMERO 51

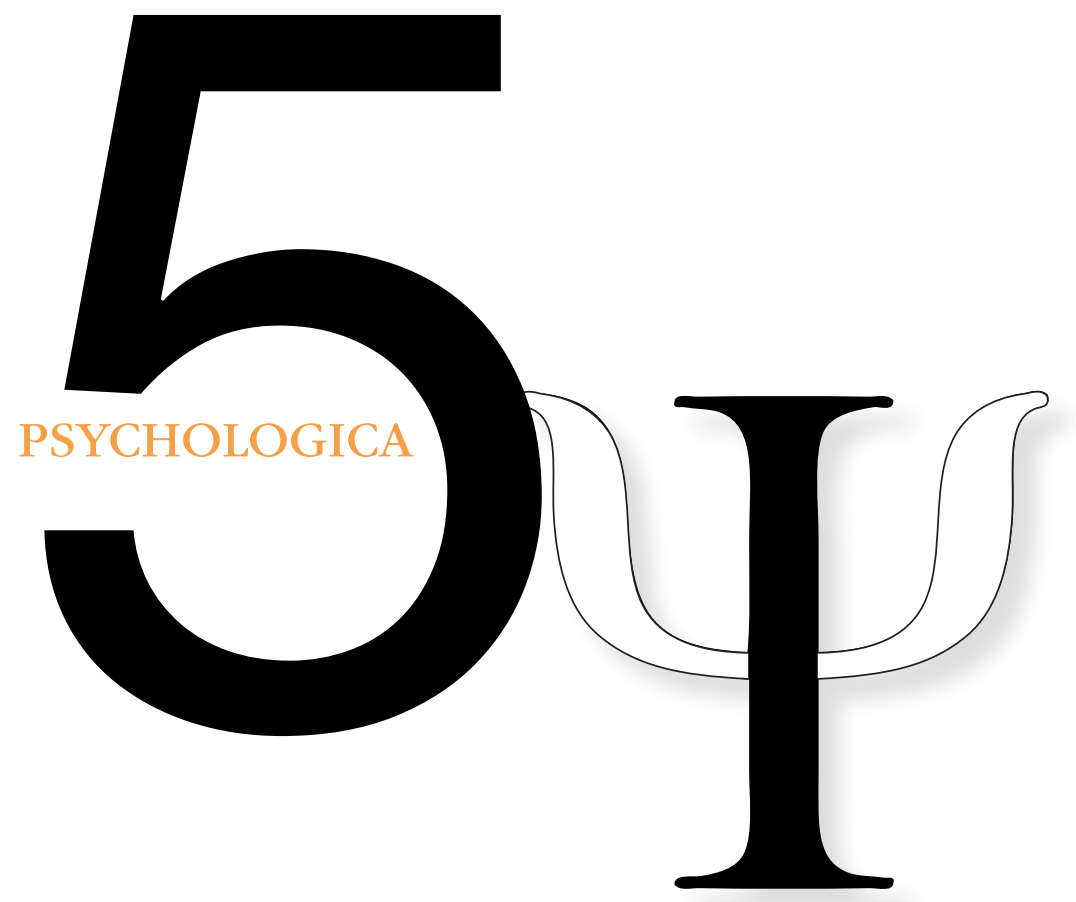

IMPRENSA DA UNIVERSIDADE DE COIMBRA

FACULDADE DE PSICOLOGIA E DE CIÊNCIAS DA EDUCAÇÃO DA UNIVERSIDADE DE COIMBRA 


\title{
Medindo as percepções de auto-eficácia no âmbito da aprendizagem e da carreira: Introdução da Secção Temática
}

\author{
José M. Tomás da Silva'
}

\begin{abstract}
Este trabalho é uma introdução da Secção Temática da Psychologica intitulada "Medindo as percepções de auto-eficácia no âmbito da aprendizagem e da carreira". Depois de discutirmos, brevemente, o conceito de auto-eficácia e qual o seu papel e relevância na explanação do funcionamento psicossocial humano, procede-se a uma apresentação sumária das cinco contribuições integradas nesta colectânea de estudos.
\end{abstract}

PALAVRAS-CHAVE: Auto-eficácia (medidas); Contextos vocacional e educativo.

O conceito de auto-eficácia, introduzido há mais de trinta anos no vocabulário das ciências psicológicas (Bandura, 1977), continua até aos nossos dias a atrair a atenção de um vasto grupo de investigadores provenientes de múltiplas áreas das ciências sociais e do comportamento.

A teoria da auto-eficácia é uma componente fundamental da teoria sociocognitiva de Bandura (1986), que por sua vez é uma extensão da sua teoria, mais antiga, de aprendizagem social. A teoria sociocognitiva de Bandura apresenta uma série de pressuposições gerais, ou assunções prévias, acerca do funcionamento psicossocial. Segundo Lent \& Maddux (1997), elas podem sumariar-se através dos seis pontos seguintes: (1) as pessoas possuem capacidades de representação simbólica da realidade (interna e externa) que as habilitam ao exercício de aprendizagens por vias indirectas (e.g., aprendizagem vicariante, ou baseada na observação de outrem) e à auto-reflexão; (2) grande parte do comportamento é intencional ou dirigido para metas (objectivos) e, portanto, é planeado e premeditado; (3) as pessoas são capazes de auto-regulação através do controlo directo do seu próprio comportamento e/ou da escolha ou modificação dos ambientes/contextos em que se inserem; As pessoas adoptam os seus próprios padrões, ou critérios, de comportamento e, dessa forma, criam os seus próprios incentivos que orientam/ 
motivam o seu comportamento futuro; (4) o funcionamento psicossocial é plurideterminado e, por isso, será sempre redutor pretender basear as explicações do comportamento num único conjunto de factores. De facto, para Bandura (1986) os acontecimentos ambientais, os factores pessoais e o comportamento interagem reciprocamente e inter influenciam-se mutuamente (modelo de causalidade recíproca triádica); (5) a teoria sociocognitiva preocupa-se explicitamente com o papel que as práticas de socialização relativas ao papel de género e as influências sócio culturais têm no comportamento; e (6) a psicoterapia e outras formas de intervenção dirigidas para a modificação do comportamento são em primeiro lugar e antes de mais, situações sociais.

As crenças, ou percepções, de auto-eficácia, que constituem uma componente chave da teoria sociocognitiva, devem a sua génese às imensas capacidades de representação simbólica dos seres humanos as quais, por sua vez, são incontornáveis para o desenvolvimento do pensamento auto-referencial. Para Bandura (1986) a percepção de auto-eficácia é definida como "os julgamentos que as pessoas fazem acerca das capacidades que detêm para organizar e executar os cursos de acção requeridos para atingir os tipos de performances designados" (p. 391).

Após várias décadas de investigação sobre as percepções de eficácia pessoal são inúmeros os estudos empíricos que se têm debruçado sobre o papel que o construto detém no funcionamento psicossocial, nos mais diversificados domínios em que este pode decompor-se, como está amplamente documentado no livro de Bandura (1997), intitulado Self-efficacy: the exercise of control. A esmagadora maioria dos estudos até agora realizados e publicados atestam a robustez do construto de auto-eficácia e a sua potencialidade explicativa do funcionamento nos planos cognitivo, da saúde, da clínica, do desporto, e das organizações (vide, por exemplo, Bandura, 1997; Maddux, 1995).

Embora inicialmente o conceito de auto-eficácia tenha sido aplicado a situações de tipo clínico (e.g., fobias, vide Bandura, Adams, \& Beyer, 1977), desde cedo captou também a atenção dos investigadores das áreas da educação e do comportamento vocacional. A bibliografia internacional respeitante aos dois domínios referidos é extensa e, em termos gerais, corrobora o importante impacto que as crenças de auto-eficácia têm na mediação e explicação do comportamento académico e vocacional (e.g., Bandura, 1993; Hackett \& Betz, 1995; Lent \& Hackett, 1987; Schunk, 1995).

Em Portugal, à semelhança do que ocorre no resto do mundo, é já significativo o número de autores que se têm dedicado a aprofundar o conhecimento existente sobre o efeito que as crenças de eficácia pessoal desempenham no funcionamento psicossocial nos domínios da saúde, da educação e do comportamento vocacional. 
A recensão dos trabalhos realizados no nosso país é uma tarefa que ainda está por fazer, embora a concretização de um projecto desse tipo pudesse constituir, indiscutivelmente, uma mais-valia para o aprofundamento e a expansão da investigação no futuro sobre a problemática em apreço.

Nesta Secção Temática da Psychologica reúnem-se cinco trabalhos especificamente dirigidos para a avaliação de crenças de auto-eficácia nos contextos de aprendizagem e do comportamento vocacional. Trata-se, na verdade, de dar à estampa as comunicações orais que integraram um Simpósio efectuado no âmbito da $15 t$ International Conference of Psychology and Education e que teve lugar de 26 a 28 de Março, de 2009, na Universidade da Beira Interior. Com a presente publicação pretende-se alcançar uma divulgação mais ampla dos trabalhos aí apresentados e, desta forma, contribuir para a literatura que procura esclarecer o papel da auto-eficácia no funcionamento humano nas áreas específicas do desenvolvimento vocacional e da aprendizagem em contextos educativos, quer tradicionais, quer não tradicionais.

O denominador comum ao conjunto dos trabalhados que integram esta secção temática, para além, evidentemente, de todos glosarem a problemática das percepções de auto-eficácia, encontra-se também na preocupação que tivemos de reunir trabalhos que se debruçassem exclusivamente sobre a medição deste construto psicossocial. A raison d'être deste enfoque funda-se na necessidade que ainda sentimos, enquanto falantes da língua Portuguesa, de dispormos de instrumentos de medida psicológicos, em número e abrangência suficiente, que nos permitam recolher dados fidedignos e válidos sobre os fenómenos comportamentais que pretendemos investigar empiricamente. Esta necessidade não é, obviamente, uma característica exclusiva da investigação no campo da auto-eficácia, porém, aqui ela assume contornos que requerem da parte dos autores, porventura, uma atenção mais reflectida. De facto, como salientou Bandura (1986), o papel da auto-eficácia no funcionamento humano será melhor clarificado com o recurso a uma "abordagem micro-analítica através da qual o pensamento auto-referente é medido em termos de auto-percepções particularizadas de eficácia, as quais podem variar segundo as actividades e as circunstâncias, em vez de, à maneira de uma disposição generalizada, avaliada através de um teste de tipo enciclopédico" (p. 396). Neste pressuposto, os instrumentos relativos às auto-percepções "devem ser feitos à medida para o domínio do funcionamento psicológico que está a ser explorado" (p. 396).

As percepções de auto-eficácia não são traços, ou disposições estáveis da personalidade, pelo contrário é mais correcto perspectivá-las como um sistema multidimensional, dinâmico e flexível de crenças respeitantes a distintos domínios de actividades e comportamentos. Para Bandura (1997), não há um sentimento 
de auto-eficácia global, mas antes percepções de eficácia pessoal em domínios específicos. No limite, este ponto de vista metodológico acerca do modelo apropriado de medida da auto-eficácia, conduz a que os investigadores interessados em estudar diferentes áreas do funcionamento psicológico tenham de construir instrumentos personalizados para cada uma das referidas áreas de funcionamento que desejam explorar. Esta é, de facto, a via mais adequada de proceder no que diz respeito à avaliação das percepções de auto-eficácia e para ajudar os investigadores a construírem boas medidas deste construto foram desenvolvidas algumas linhas de orientação que, naturalmente, devem ser consultadas por todos quantos desejem elaborar medidas de auto-eficácia (cf., Bandura, 1997 [cf., especialmente, pp. 42-54], 2006; Lent \& Brown, 2006; Pajares, Hartley, \& Valiante, 2001).

Embora a construção "caseira" de medidas de auto-eficácia seja em algumas situações a única via em aberto para o investigador, felizmente que hoje em dia existem outras alternativas mais expeditas como, por exemplo, usar uma medida já desenvolvida por outro autor. Assim, no sitio da Internet http://www.des.emory. edu/mfp/self-efficacy.html encontramos vários exemplos de escalas pertencentes a distintos domínios psicossociais, desenvolvidas por uma comunidade internacional de investigadores interessados no estudo da auto-eficácia.

Com a colectânea de estudos incluídos nesta Secção Temática é nossa intenção contribuir para tornar mais diligente o processo de investigação da auto-eficácia nos domínios anteriormente referidos, ao apresentar cinco instrumentos deste construto e as suas principais propriedades psicométricas, em termos de garantia, ou precisão, e de validade.

No primeiro trabalho Paulo Cardoso (Universidade de Évora) e Isabel do Vale (Escola Básica Integrada de Patrício Prazeres, Lisboa) apresentam as principais propriedades de medida de um instrumento inovador destinado a avaliar as crenças de auto-eficácia a respeito dos papéis de carreira (e.g., Serviços à Comunidade, Casa/Família, Tempos Livres, Estudante e Trabalhador). As análises das respostas recolhidas em duas grandes amostras de alunos dos $9^{\circ}$ e $12^{\circ}$ anos de escolaridade revelam bons índices de consistência interna e de validade para as diferentes facetas de auto-eficácia incluídas na escala. Os autores demonstraram que é exequível medir a auto-eficácia de adolescentes a respeito de distintos papéis de carreira e sugerem, adicionalmente, algumas possibilidades de integrar a informação recolhida com o seu instrumento no aconselhamento e na investigação do desenvolvimento de carreira.

No artigo seguinte, José Tomás da Silva, Maria Paula Paixão e Ana Margarida Albuquerque, da Universidade de Coimbra, apresentam alguns dados originais sobre as características psicométricas da adaptação à língua Portuguesa de um dos 
instrumentos mais conhecidos internacionalmente da auto-eficácia de carreira, a Career decision Self-Efficacy Scale (CDSES). A CDSES avalia as crenças de auto-eficácia dos respondentes a respeito de diferentes competências implicadas na tomada de decisão de carreira (e.g., Auto-Avaliação, Informação Ocupacional, Selecção de Objectivos, Planeamento e Resolução de Problemas). Os dados psicométricos recolhidos numa amostra de conveniência de alunos do $9^{\circ}$ ano de escolaridade revelam que as respostas nas sub-escalas apresentam níveis de precisão aceitáveis, embora o resultado para o somatório dos itens (escala completa) seja bastante mais fiável, ou preciso. Consistentemente com os resultados prévios de estudos internacionais, os autores, recorrendo a uma análise de componentes principais, não encontraram evidências a favor do modelo estrutural com cinco dimensões postulado para os itens da escala. Nesse sentido sugerem que, provavelmente, a CDSES mede fundamentalmente uma crença generalizada de auto-eficácia a respeito da tomada de decisão de carreira.

No terceiro estudo desta Secção Temática, Maria Odília Teixeira (Universidade de Lisboa), revisita uma série de dados provenientes de vários estudos empíricos que a autora e colaboradores têm vindo sistematicamente a recolher com a versão Portuguesa de um instrumento desenvolvido por Albert Bandura e intitulado Multidimensional Scales of Perceived Self-Efficacy. Os resultados das investigações que até agora têm sido realizados revelam, como é referido pela autora, que as medidas das diferentes facetas (sub-escalas) apresentam bons indicadores de consistência interna nas distintas amostras observadas. Os resultados obtidos com amostras de estudantes do ensino básico, secundário e superior suportam a utilidade da medida, tanto na investigação como na intervenção psicológica nos contextos de aprendizagem e de carreira, bem como na análise das suas recíprocas influências.

No quarto estudo Albertina Lima Oliveira (Universidade de Coimbra) revisita alguns estudos prévios e apresenta os resultados de novos trabalhos com a versão Portuguesa do Self-Efficacy for Self-Directed Learning Questionnaire. Em particular, a autora neste artigo expande o campo de aplicação do questionário à população de adultos que se encontram envolvidos em processos de Reconhecimento, Validação e Certificação de Competências (RVCC). As características psicométricas analisadas em distintas amostras de respondentes, designadamente no que respeita à consistência interna e à validade dos resultados, revelaram-se bastante adequadas e atestam a robustez e utilidade do instrumento como medida da auto-eficácia para a autodirecção da aprendizagem de indivíduos adultos.

Encerra esta colectânea de estudos sobre a avaliação da auto-eficácia um trabalho de Rute David, Maria Paula Paixão e José Tomás da Silva, da Universidade de Coimbra, sobre a relação entre os interesses vocacionais e as competências percebidas 
(percepções de eficácia pessoal) em crianças e jovens adolescentes. Neste estudo transversal de alunos do Ensino Básico ( $3^{\circ}, 6^{\circ}$ e $9^{\circ}$ anos de escolaridade), os autores examinam, especialmente, as propriedades de medida da versão Portuguesa do Inventory of Children's Activities - Revised, um instrumento baseado na teoria dos tipos de personalidade e dos ambientes profissionais de John Holland. Dada a inexistência de instrumentos devidamente adaptados para a investigação do comportamento e do desenvolvimento vocacional das crianças, acreditamos que esta nova medida contribua para o incremento e aprofundamento da investigação futura com sujeitos de grupos etários mais novos.

Os cinco estudos incluídos nesta Secção Temática proporcionam uma boa imagem, ainda que inevitavelmente incompleta, da vitalidade da investigação que é realizada no nosso país na área da auto-eficácia a plicada aos domínios do funcionamento educativo e vocacional. Esperamos que a colectânea de estudos agora dados à estampa possam servir de incentivo à realização de novas investigações sobre o impacto das percepções de auto-eficácia no funcionamento psicossocial das pessoas.

\section{Referências bibliográficas}

Bandura, A. (1977). Self-efficacy: Toward a unifying theory of behavioral change. Psychological Review, 84, 191-215.

Bandura, A. (1986). Social foundations of thought and action: A social cognitive theory. Englewood Cliffs, NJ: Prentice-Hall.

Bandura, A. (1993). Perceived self-efficacy in cognitive development and functioning. Educational Psychologist, 28, 117-148.

Bandura, A. (1997). Self-efficacy: The exercise of control. New York: Freeman.

Bandura, A. (2006). A guide to the construction of self-efficacy scales. In F. Pajares \& T. Urdan (Eds.), Self-efficacy beliefs of adolescents, (Vol.5., pp. 307-337). Greenwich, CT: IAP - Information Age Publishing.

Bandura, A., Adams, N. E., \& Beyer, J. (1977). Cognitive processes mediating behavior change. Journal of Personality and Social Psychology, 35, 125-139.

Hackett, G. \& Betz, N. E. (1995). Self-efficacy and career choice. In J. E. Maddux (Ed.), Self-efficacy, adaptation, and adjustment: Theory, research, and application (pp. 249-280). New York: Plenum Press.

Maddux, J. E. (Ed.) (1995). Self-efficacy, adaptation, and adjustment: Theory, research, and application. New York: Plenum Press.

Lent, R. W. \& Brown, S. D. (2006). On conceptualizing and assessing social cognitive constructs in career research: A measurement guide. Journal of Career Assessment, 14, 12-35.

Lent, R. W. \& Hackett, G. (1987). Career self-efficacy: Empirical status and future directions. Journal of Vocational Behavior, 30, 347-382.

Lent, R. W. \& Maddux, J. E. (1997). Self-efficacy: Building a sociocognitive bridge between social and counseling psychology. The Counseling Psychologist, 25, 240-255. 
Pajares, F., Hartley, J., \& Valiante, G. (2001). Response format in writing self-efficacy assessment: Greater discrimination increases prediction. Measurement and Evaluation in Counseling and Development, 33, 214-221.

Schunk, D. H. (1995). Self-efficacy and education and instruction. In J. E. Maddux (Ed.), Self-efficacy, adaptation, and adjustment: Theory, research, and application (pp. 281-303). New York: Plenum Press.

\section{Mesurer la perception de l'auto-efficacité dans les contextes d'apprentissage et de carrière: Introduction à la section thématique}

Ce travail est une introduction à la section thématique de Psychologica intitulée «Mesurer la perception de l'auto-efficacité dans les contextes d'apprentissage et de carrière». Après avoir discuté brièvement le concept sentiment d'efficacité personnelle et quel est leur rôle et la pertinence dans l'explication du fonctionnement psychologique humain, les cinq articles de ce recueil d'études sont présentées de façon concise.

MOTS-CLÉS: Efficacité personnelle (mesures); Contextes vocationnelle et éducatif.

\section{Measuring perceptions of self-efficacy within learning and career contexts: Introduction to the thematic section}

This work is an introduction to the thematic section of Psychologica entitled "Measuring perceptions of self-efficacy in learning and career contexts." After discussing briefly the concept of self-efficacy and what is their role and relevance in explanation of human psychosocial functioning, the five articles included in this collection of studies are concisely presented.

KEY-WORDS: Self-efficacy (measures); Vocational and educational contexts. 